\title{
Novedades Nomenclaturales en "Tipos Grisebachianos"
}

\author{
LUIS ARIZA ESPINAR ${ }^{1}$
}

\begin{abstract}
Summary: Nomenclatural novelties in types from Grisebach. The study of Grisebach's types from CORD has allowed clarifying the identity of two dubious or not well known names. A new combination in Byttneria is also proposed.
\end{abstract}

Key words: Argentina, Grisebachian types, Byttneria.

Resumen: El estudio de tipos Grisebachianos de CORD ha permitido aclarar la identidad de dos nombres dudosos o no bien conocidos. Se realiza una nueva combinación en Byttneria.

Palabras clave: Argentina, tipos Grisebachianos, Byttneria.

A raíz de mi participación en la labor de procesamiento de datos de los "tipos" del Herbario CORD, principalmente los "grisebachianos", en relación al Proyecto Latin American Plant Initiative (LAPI), han surgido novedades, algunas de las cuales se dan a conocer aquí.

Se ha dispuesto de los materiales duplicados de la colección de Lorentz \& Hieronymus de Argentina y Bolivia que estudiara Grisebach, como así también de fotografías de los holotipos depositados en Goettingen (GOET). De gran valor han resultado también las observaciones que sobre éstos realizara A. T. Hunziker, y que figuran en una versión inédita de su Catálogo de los Tipos Grisebachianos que publicara en 1960.

La presente contribución trata de nombres que, por algún motivo, hoy en día están considerados "nombres dudosos" o de identidad desconocida, tal vez por no haber sido accesibles los materiales correspondientes a los autores que se han ocupado, en su momento, de los respectivos géneros. Para este propósito, se ha consultado la información disponible en TROPICOS (www.mobot.org) y Catálogos de la Flora Argentina (Zuloaga \& Morrone, 1999) y del Cono Sur ( Zuloaga et al., 2008), como se indica en cada caso.

\footnotetext{
1 Instituto Multidisciplinario de Biología Vegetal-Museo Botánico de Córdoba (CONICET-UNC). C.C. 495, 5000. Córdoba. E-mail: arizaesp@imbiv.unc.edu.ar
}

\section{NOMBRES DUDOSOS O NO BIEN CONOCIDOS}

\section{¿Qué es Alternanthera pulchella var. ciliata} Griseb.?

Variedad descripta por Grisebach (1874: 36), que ha pasado desapercibida por quienes se han ocupado de Amaranthaceae argentinas (Pedersen, 1999). No obstante, Suessenguth (1934: 304) ya alertó que ese nombre grisebachiano pertenecía a una especie de Paronychia Mill. Hunziker (1960: 310) consignó que no encuentra en CORD material referible a este nombre pero, cuando estudió el material de GOET, encontró el original, anotó sus datos, y ubicó más tarde, en CORD, el duplicado correspondiente. El isotipo en nuestro Herbario es un excelente ejemplar, mientras que la foto del holotipo, en CORD, muestra varios trozos pequeños de plantas, $\mathrm{y}$, en un fragmento del mismo, también en CORD, se puede advertir mezcla. Nuestro isotipo concuerda bastante bien con material de Paronychia communis Cambess. (se siguió para este nombre el criterio de Volponi, 1998). En el fragmento de holotipo antes citado, puede observarse, además, una ramita con hojas elípticas, glabras, similares a las de P. hieronymi Pax. Luego:

Paronychia communis Cambess., Fl. Bras. Merid. (quarto ed.) 2: 186. 1829 (Isotipo: BRAZIL, St. Hilaire 1564, MPU). 
= Alternanthera pulchella var. ciliata Griseb., Pl. lorentz.: 36, 1874 . Tipo: ARGENTINA. Prov. Tucumán: Cuesta de Casilla, P. G. Lorentz 769, 5-IV1872. Holotipo GOET; isotipo: CORD 00004833!; fragmento de holotipo y fototipo: CORD!

\section{¿Qué es Machaerium pseudotipa Griseb.?}

Este nombre grisebachiano no figura en las recientes contribuciones de Ulibarri (1997), Zuloaga \& Morrone (1999), Zuloaga et al. (2008). Sin embargo, está citado en TROPICOS y en Index Kewensis (aunque como "pseudotipe"). Merece destacarse que este hecho no pasó desapercibido al ojo avezado de Burkart (1952: 228) quién al referirse a las especies de Machaerium dijo “... M. pseudotipa Griseb. en cambio es Pterogyne nitens". La propuesta formal de la sinonimia es la siguiente:

Pterogyne nitens Tul., Ann. Sci. Nat. Bot. Sér. 2, 20: 140. 1843.

Machaerium pseudotipa Griseb., Symb. Fl. Argent::110. 1879. Tipo: ARGENTINA. Prov. Salta: Luna Muerta, entre Dragones y Orán, $P$. G. Lorentz \& G. Hieronymus 652, 30-VIII-1873 "palo mortero" (Holotipo GOET, isotipo: CORD 00005833!).

Obs. I . El holotipo, en GOET, lleva estos datos: Salta: Bei Luna Muerta an Bermejo, 30-VIII-1873 y 4 renglones indescifrables, fide Hunziker (inédito).

Obs. II. El segundo ejemplar que Hunziker (op. cit.: 323) citara como probable sintipo (Salta. Dragones, entre Orán y Gran Chaco, cerca de la Luna Muerta, med. VIII, 1873, P. G. Lorentz \& G. Hieronymus 587), tal vez no fue visto por Grisebach, pues no pudo ubicarlo en GOET. Por otro lado, el paratipo coleccionado en Paraguay: L'Asomption, Balansa 2540, Avril 1874, solo existe en GOET.

Obs. III. Resulta interesante que el nombre vulgar anotado por Grisebach (palo mortero) y que también fue citado por Burkart (l.c.), no figura en la contribución de Ulibarri (1997).

\section{UNA NUEVA COMBINACIÓN EN Byttneria (Sterculiaceae)}

En 1879, Grisebach describió a Caperonia acalyphifolia en base a un material coleccionado en Bolivia. Este nombre no ha sido aclarado por quienes se han ocupado de este género en el país ( $\mathrm{O}^{\prime}$ Donell \& Lourteig, 1942), mientras que Catálogos actuales (Zuloaga \& Morrone, 1999; Zuloaga et al., 2008) le asignan "validez desconocida" (aunque la cita original era para Bolivia). Tal vez el problema ha sido que el material tipo, tanto el de GOET como el de CORD son muy pobres pues solo tienen restos florales que no permiten una fácil identificación. Sin embargo, Pax (1912: 49) en su revisión de Caperonia excluye a este binomio del género, aunque anota la combinación de Kuntze de 1894: Argythamnia acalyphifolia (Griseb.) Kuntze, pero advirtiendo que es una Büttnera. Llama la atención que este dato haya pasado inadvertido, tal vez por lo expresado antes sobre la pobreza del material. Al revisar los tipos grisebachianos en CORD, pude observar, en un sobre, al pie de la cartulina, la existencia de 3 frutos ("cocos"), similares a los señalados para Byttneria. Conociendo a Byttneria tucumanensis por haberla coleccionado en más de una vez, pude analizar el tomento de pelos estrellados, tallos acanalados con acúleos retrorsos y morfología de los frutos, de ambos materiales, y de este análisis, no me quedaron dudas sobre la identidad de esta Byttneria con el material del binomio de Grisebach, razón por la cual propongo la siguiente combinación y sinonimia.

Byttneria acalyphifolia (Griseb.) Ariza comb. nov.

Caperonia acalyphifolia Griseb., Symb. Fl. Argent.: 58. 1879. Tipo: BOLIVIA. Cuesta de Agurenda, entre Itaperenda y Jucaiva, $P$. G. Lorentz \& G. Hieronymus 621, 21-VII-1873. Holotipo: GOET, isotipo CORD 00005967!. Foto del holotipo, CORD.

Buettneria hirsuta var. parvifolia Kuntze, Revis. Gen. Pl. 3 (3): 24. 1898. (Fototipo, NY !).

Byttneria tucumanensis Cristóbal, Bonplandia 4: 211. fig. 52. 1976. Tipo: ARGENTINA. Prov. Tucumán: Graneros Dique San Ignacio, La Cocha, 10-II-1964, A. Krapovickas \& C. Cristóbal 11275 (Holotipo: CTES; isotipo: CORD 00003324!); Prov. Córdoba. Dpto. Colón: Rio Ceballos, inmediaciones de Pozos Verdes, 22-III- 1969, L. Ariza Espinar 2369 (Paratipo: CORD 00003325!). 
Obs. I. El holotipo de Caperonia acalyphifolia presenta restos florales y un sobre del cual desconozco su contenido. Según Hunziker, la fecha del mismo es: 21-VI-1873 ( en vez de VII, tal vez error del copista) y solo dice "Cuesta de Aguairenta" y le falta "entre Itaperenda y Jucaiva".

Obs. II. Las descripciones, tanto la de Grisebach como la de Cristóbal (1976) son muy completas, incluso la lámina de B. tucumanesis es excelente, razón por la cual no amerita que la misma deba ser repetida.

Obs. III. Conviene destacar la observación de Cristóbal sobre la caducidad de la capa superficial de los cocos porque ello también es muy evidente en los 3 cocos observados en el isotipo de Grisebach.

\section{Conclusiones}

La revisión de los tipos grisebachianos me ha permitido aclarar algunos de los binomios considerados "nombres dudosos" o "desconocidos" al disponer de los isotipos depositados en CORD. Me han sido de gran ayuda las anotaciones que Hunziker realizara durante su estada en el Herbario de Goettingen sobre los originales estudiados por Grisebach y que éste condensara en sus dos obras magistrales tales como Symbolae ad Floram argentinam y Plantae Lorentzianae. Así, pues: Alternanthera pulchella var. ciliata Griseb. es Paronychia communis Cambess. y Machaerium pseudotipa un sinónimo de Pterogyne nitens Tul. Por otro lado Caperonia acalyphifolia Griseb. pertenece al género Byttneria, razón por la cual se realiza la respectiva combinación.

\section{Agradecimientos}

A la Dra. Gloria Barboza por la lectura crítica del presente manuscrito.

\section{Bibliografía}

BURKART, A. 1952. Las Leguminosas Argentinas. Silvestres y Cultivadas, I- XV. 1- 569. Acme Agency, Buenos Aires.

GRISEBACH, A. 1874. Plantae Lorentzianae. Göttingen.

GRISEBACH, A. 1879. Symbolae ad Floram argentinam. Göttingen.

HUNZIKER, A. T. 1960. Catálogo de los tipos "grisebachianos" conservados en Córdoba. Bol. Acad. Nac. Ci. 41: 283-421.

INDEX KEWENSIS. 1996. 2nd. ed. CD-rom.

O’ DONELL, C. A. \& A. LOURTEIG. 1942. Chrozophoreae Argentinae. Lilloa 8: 37-81.

PAX, F. 1912. Euphorbiaceae-AcapypheaeChrozophorinae. In: A. Engler, Das Pflanzenreich 57 (IV.147.VI): 1-142.

PEDERSEN, T. M. 1999. Amaranthaceae. En: F. O. Zuloaga \& O. Morrone (eds.), Catálogo de las Plantas Vasculares de la Argentina II. Monogr. Syst. Bot. Missouri Bot. Gard. 74: 16-41.

SUESSENGUTH, K. 1934. Neue und kritische Amarantaceen aus Süd- und Mittel-America. Repert. Spec. Nov. Regni Veg. 35: 298-337.

ULIBARRI, E. A. 1997. Tribu Caesalpinieae. En: A. T. Hunziker (ed.), Flora Fanerogámica Argentina 32: 6-26. PROFLORA, Córdoba.

VOLPONI, C. R. 1998. Caryophyllaceae. En: L. Novara (dir.), Aportes Bot. Salta, Ser. Fl. 5: 1-52.

ZULOAGA, F. O. \& O. MORRONE (eds.). 1999. Catálogo de las Plantas Vasculares de la República Argentina. Monogr. Syst. Bot. Missouri Bot. Gard. 74: 1-1269.

ZULOAGA, F. O., O. MORRONE \& M. J. BELGRANO (eds.). 2008. Catálogo de las Plantas Vasculares del Cono Sur. Monogr. Syst. Bot. Missouri Bot. Gard. 107 (2/3): 985-3348.

Recibido el 21 de noviembre de 2012, aceptado el 18 de febrero de 2013. 
